\title{
EXPANSIÓN DE LA ACTIVIDAD LIMONERA Y REPERCUSIONES SOBRE LOS PEQUEÑOS PRODUCTORES EN LA PROVINCIA DE TUCUMÁN (ARGENTINA)
}

\author{
ANA ESTER BATISTA \\ JUAN JOSÉ NATERA RIVAS
}

\begin{abstract}
RESUMEN
El objetivo de la presente contribución es mostrar las características generales de la activiclad limonera en la provincia de Tucumán (Argentina), prestando especial atención a los pequeños productores. Evolución del tamaño de las explotaciones, mercados o costos de produccion son aspectos que abordaremos, comparándolos, en algún caso, con la situación de las explotaciones españolas.
\end{abstract}

\section{ABSTRACT}

The aim of this paper is show the general characters or the citrus activity at Tucumán (Argentina), focused on the small productors. The evolution of the explotation's size, markets or productions costs are studied, and compared, in some cases, with the spanish ones.

\section{INTRODUCCIÓN.}

La actividad citrícola, y dentro de ella la limonera, tiene una fuerte implantación en la provincia de Málaga, especialmente en el área del Valle del Guadalhorce. En la actualidad contamos con un conjunto de investigaciones en las que se abordan diferentes aspectos de la misma (Larrubia 1988, 1994, 1996, Ocaña y Larrubia 1993), conformando un cuadro muy completo de sus principales características y vicisitudes. Es por ello que creemos que puede ser de alguna utilidad mostrar, aunque sólo sea de una forma muy general, los caracteres que esta misma actividad presenta en un área geográfica diferente, la provincia de Tucumán (Argentina).

En ella, y en un contexto económico muy diferente al español, las fuerzas del mercado actúan libremente, lo que ha derivado en que determinados estratos de productores hayan quedado marginados en el camino que ha llevado a Tucumán a convertirse en una de las más importantes zonas productoras e industrializadoras de limón a nivel mundial. En este marco, y como a lo largo del texto mostraremos, la pervivencia de las explotaciones de menor tamaño, posible en España gracias a mecanismos de protección, resulta impensable en el área tucumana, dado que producir fruta de calidad y a precios competitivos (única vía segura para la supervivencia de la explotación) tan sólo está al alcance de unos pocos; aquellos que no lo logran, y 
ante la ausencia de mecanismos de protección, simplemente se ven obligados a abandonar progresivamente la actividad.

\section{BREVE RESEÑA HISTÓRICA DE LOS CÍTRICOS EN ARGENTINA.}

Aunque la superficie dedicada a los cítricos en Argentina (introducidos por los jesuitas a fines del XVI) comenzó a extenderse a finales del siglo pasado, ha sido en éste cuando su crecimiento ha sido mayor. En un primer momento el cultivo de naranja se resolvía como mayoritario, seguido en importancia por la mandarina, el limón y el pomelo, particularidad que caracterizaba a las diferentes regiones citrícolas del país. Estos productos tenían como destino el mercado local, debiclo a las dificultades existentes en lás vías de comunicación y los altos costos de transporte para poder colocar la producción en Buenos Aires, principal mercado consumidor del país.

Más tarde, y al tiempo que se extendía la superficie citrícola, las diferentes regiones se fueron orientando hacia una especializacón productiva por especie, como consecuencia de la interacción de un conjunto de factores, entre los que es importante resaltar las condiciones agroecológicas, exigencias y cercanía de los mercados, vías de transporte, capital y tecnología disponibles, y, en no menor medida, la incidencia de las enfermedades en las plantaciones. Como resultado de ello hoy encontramos en la región litoral (Misiones, Corrientes, Entre Ríos) y la pampa un predominio de cultivo de naranjas, mientras que en el Noroeste Argentino la situación es más dispar; así, Jujuy y Salta se especializan en la producción de naranjas (a las que se une el pomelo en esta última provincia), mientras que en Tucumán el rol central lo ocupa el limón.

En el pidemonte tucumano durante la década del '60 las plantaciones con naranja se vieron afectadas por la podredumbre de raicillas (enfermedad que exterminó la mayor parte de ellas); a partir de este momento la producción se orientó al cultivo del limón por ser resistente a esta enfermedad. Hasta ese momento, las explotaciones tenían un carácter nétamente familiar; se trataba de inmigrantes que habían llegado en las primeras décadas de este siglo, que, tras un periodo de arrendamiento con dedicación al cultivo de hortalizas, el hecho de acceder a la propiedad de las tierras les había permitido orientarse al cultivo de los cítricos. No obstante, descle la década del setenta, la implantación de nuevas plantaciones de limones vino acompañada por emprendimientos industriales destinados al procesamiento de una cosecha de limón que, especialmente la de invierno, era en gran parte desarpovechada. A partir de una industrialización casi artesanal, en la actualidad se ha llegado a una situación en la que, tras la apertura de mercados en el ámbito internacional, la provincia de Tucumán es, por sí sóla, el área mundial en la que mayor cantidad de limón se destina para industria, por encima de países como Estados Unidos. El valor de las exportaciones, tanto de fruta fresca como de derivados industriales en 1996 fue de 194 millones de dólares, que junto al volumen de fruta producido (700.000 tns. en ese mismo año) dan cuenta de las profundas transformaciones que el sector ha experimentado.

Como consecuencias de dichas transformaciones, son los pequeños productores los que se han visto expulsados de la misma, desde el momento en que al tratarse de una actividad 
altamente competitiva, en la que los precios obtenidos están en relación directa con la calidad del producto, y al no contar con medidas destinadas a proteger a los estratos peor situados dentro de la escala productiva, se ha registrado un importante descenso en las explotaciones de menor tamaño, imposibilitadas para continuar con la actividad.
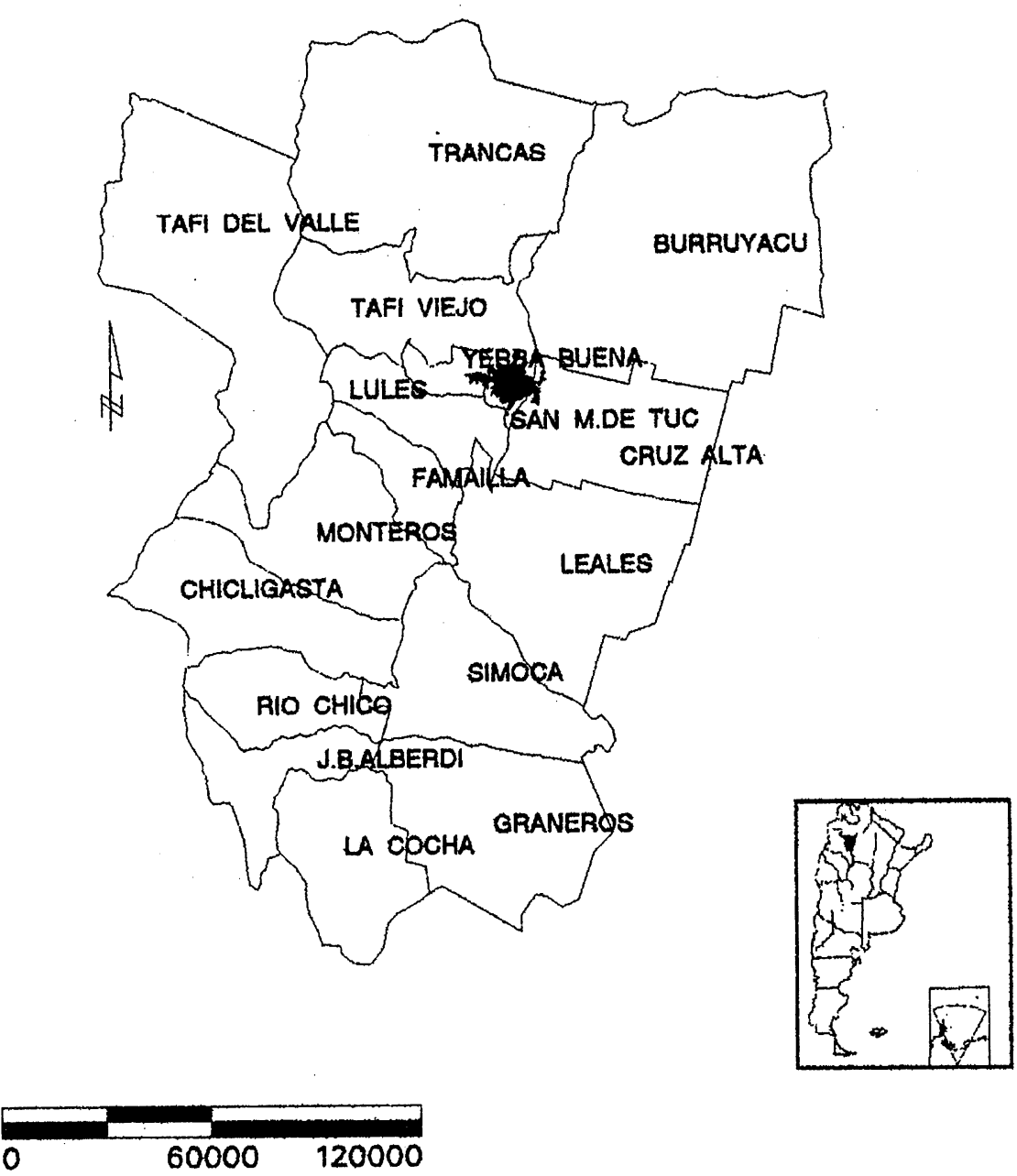

Base cartográfica elaborada por el I.E.G. do la U.N.T.

Figura 1

Localización y división administrativa de la provincia de Tucumán (Argentina) 


\section{LA EVOLUCIÓN DE LA ESTRUCTURA DE LAS EXPLOTACIONES LIMONERAS TUCUMANAS.}

La superficie cledicada al cultivo del limón en la provincia de Tucumán ha experimentado un importante incremento desde finales de los años sesenta, pasando de las aproximadamente 6.800 has. de $1969^{\prime}$ a las 23.363 de 1995 ; este proceso de incremento se ha acelerado especialmente en el periodo 1990/91-1995/96 (último para el que tenemos datos), dado que según la Secretaría de Estado de Agricultura y Ganadería -en adelante S.E.A.G.- en este lapso se implantaron 4.684 has., el $20 \%$ de la superficie limonera actual, como culminación de un proceso que se inició en los últimos años de la década de los setenta. Paralelamente se han obtenido incrementos en la productividad que permitieron pasar de las 23 tns./ha. en el 76 a las 30 de $1995^{2}$. No obstante, dicho aumento de la superficie limonera ha estado acompañado de profundas transformaciones en lo concerniente tanto al número de explotaciones como a su clistribución por estrato de tamaño.

Es así que el número de explotaciones ha descendido de manera importante en los escasos treinta años transcurridos descle el primer Censo Citrícola, dado que de las 1.002 existentes en 1969, se pasó a 626 en 1974 y a 587 en 1988, para llegar a la actualidad (1995) con tan sólo 514 explotaciones dedicadas a la actividad limonera. Pero además, este descenso ha venido acompañado por importantes variaciones en la distribución del número de explotaciones por estrato de tamaño; tal y como se aprecia en el gráfico 1, son las de más reducida extensión, inferiores a 5 has., las que han sufrido un decremento más importante (de las 632 en 1969 a las 108 de 1995), habiendo descendido también las menores de 10 has. En contraste, es en los intervalos en los que quedan encuadradas las explotaciones mayores en los que el proceso ha sido el contrario, ésto es, hemos asistido a un importante incremento en el número de explotaciones y, con toda seguridad, del número de has. ocupadas por ellas. En este sentido, es preciso indicar que no nos es posible mostrar la evolución del grado de concentración de la tierra cultivada con limón, descle el momento en que las cifras correspondientes a 1995 muestran graves deficiencias, y a que dicha información no es ofrecida en el Censo de 1969; tan sólo podemos mostrar la situación existente en 1988. En este año, las explotaciones mayores de 50 has. eran las que abarcaban la mayor parte de la superficie dedicada al limón, un 63\%, mientras que su peso en relación al número de explotaciones no llegaba al 10\%; y eran las mayores de 500 has. (cuatro en total), las que abarcaban 5.000 has. sobre las 16.000 del total provincial. En contraste, las explotaciones por debajo de las 10 has. (un 56\% del total) tan sólo suponían el 8\% de la tierra, lo que da cuenta del elevado grado de concentración de la tierra dedicada a este cítrico.

1. Estimación propia a partir de los datos de número de plantas suministrados por el Censo de Explotaciones Citrícolas de 1969; se ha considerado el empleo de un matreo de $7 \times 7 \mathrm{~ms}$. (204 plantas/ha.), el más usual en aluel
momento.

2. Fs preciso indicar que esta última cifra está subestimáala descle el momento en que un tercio de la superficie implantada con limón no ha alcanzado aún su máxima productividad por la junventud de las plantaciones
(1) irección 1997, p.9). 
Gráfico 1.

Evolución del número de explotaciones por estrato de tamaño en la Provincia de Tucumán (1969-1995).

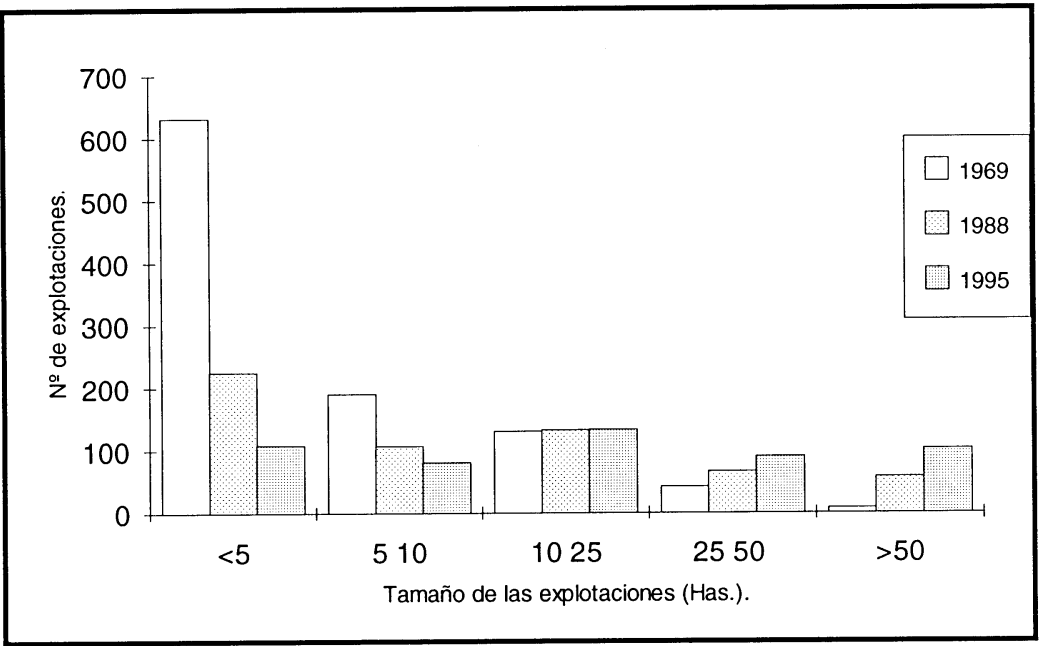

Fte: Censos de explotaciones citrícolas.

Elaboración propia.

Pero además, y dentro del marco general de incremento de la superficie dedicada al limón, disminución de la cantidad total de explotaciones y descenso del número de las más pequeñas contrastando con el aumento de las mayores, es preciso indicar que existen ciertas diferencias a nivel departamental. Así, en aquellos departamentos no incluídos en la zona citrícola tradicional se ha registrado un aumento del número de grandes explotaciones ligado a la extensión de la superficie limonera. Es el caso de Burruyacu, Chicligasta o Monteros, Lules en menor medida, departamentos en los que se centra fundamentalmente la puesta en producción de nuevas plantaciones. La tierra por ellas ocupadas proviene bien de cambios en el uso del suelo -reemplazando en las tres últimas circunscripciones al cultivo de caña de azúcar-, o por la puesta en explotación de nuevas tierras tras su desmonte, en Burruyacu.

En contraste, en aquellos departamentos en los que la actividad citrícola es más añeja, y contaban con una distribución de la propiedad mucho más atomizada, se ha registrado la formación o, más comúnmente, ampliación, de medianas propiedades (y, menos frecuentemente, grandes) a través de la compra de aquellas de más reducida extensión, en ocasiones dedicadas también a la producción de otros cítricos, con una importancia menor del proceso de 
cambio de uso del suelo. Es el caso de Famaillá, Tafí Viejo o Yerba Buena, departamentos en los que el aumento en el número de este tipo de explotaciones no ha venido acompañado de una crecimiento similar en el área limonera (tabla 1).

Tabla 1.

Variaciones del número de explotaciones por estrato de tamaño y del total de superficie dedicada al limón (1988/1995).

\begin{tabular}{lcccc}
\hline Departamento & $\begin{array}{c}\text { Menos 25 Has. } \\
(1988 / 1995)\end{array}$ & $\begin{array}{c}25 \text { y 200 Has. } \\
(1988 / 1995)\end{array}$ & $\begin{array}{c}\text { Mas de 200 Has. } \\
(1988 / 1995)\end{array}$ & $\begin{array}{c}\text { Total superficie. } \\
(1988 / 1995)\end{array}$ \\
\hline Burruyacu & $151 / 86$ & $37 / 54$ & $2 / 11$ & $3.799 / 6.346$ \\
Chicligasta & $2 / 8$ & $5 / 7$ & $0 / 2$ & $219 / 1.625$ \\
Monteros & $5 / 6$ & $0 / 10$ & $0 / 2$ & $588 / 1.946$ \\
Lules & $21 / 10$ & $7 / 16$ & $0 / 1$ & $275 / 743$ \\
Famaillá & $21 / 18$ & $11 / 18$ & $1 / 3$ & $3.081 / 3.364$ \\
Tafí Viejo & $179 / 121$ & $33 / 46$ & $6 / 7$ & $5.848 / 5.390$ \\
Yerba Buena & $31 / 28$ & $10 / 9$ & $1 / 0$ & $1.202 / 1.326$ \\
\hline
\end{tabular}

Fte: Censo citricola 1995.

Elaboración propia.

\section{CIRCUITOS COMERCIALES DEL LIMÓN TUCUMANO.}

El destino de la producción de las explotaciones limoneras tucumanas es variado, estando en relación directa con la calidad de la fruta obtenida. Así, la de mayor calidad se orienta a la comercialización como producto en fresco en el mercado internacional; no obstante, y dado el nivel de exigencia de los países importadores, la fruta debe, para ser aceptada, haber pasado previamente por una serie de labores tendentes a homogeneizar calibres y a mejorar su presentación, tales como lavado, encerado, empaquetado, etc., comercializándose generalmente en cajones de 18 kgrs. Estas labores se realizan en la propia provincia en plantas empacadoras, que se nutren de fruta proveniente tanto de sus propias explotaciones-como consecuencia de procesos de integración vertical descendente-como, cada vez en menor medida, de otros productores. Este tipo de fruta de alta calidad se obtiene generalmente en las explotaciones con más de 50 has., descle el momento en que, como más adelante mostraremos, son las que tienen capacidad económica suficiente como para realizar las inversiones necesarias para su obtención; en este sentido, y ante las exigencias de unos mínimos de calidad en la fruta por parte cle las empacadoras, todo aquel productor que no los alcance queda automáticamente excluíclo de este circuito comercial. Si a ello unimos la tendencia a evitar la salida hacia este mercado de fruta de menor calidad (siempre en el contexto de altos estándares) o de tamaños extremos (que se vende a menor precio facilitando así la aplicación de gravámenes por parte de la Unión Europea, principal destino exterior del limón tucumano), resulta evidente que la participación en las ventas en el exterior se está volviendo cada vez más exclusiva. 
La fruta que no puede ser exportada se vende como segunda opción en el mercado nacional, también en fresco; no obstante, este mercado viene sufriendo una contracción desde los años setenta, que ha supuesto el paso de las 90.000 Tns. de media destinadas a él en la década del setenta a tán sólo 54.000 en la actual. Dado que la producción se ha incrementado considerablemente, el descenso porcentual ha sido más marcado (gráfico 2). El consumo de esta fruta en fresco se halla poco desarrollado en el país, y estaría en franco descenso; así, y siguiendo a Amigo (1994, 9), si en 1980 se consumían 14,017 kgrs./hab./año, en 1986 se había bajado a 7,8 y en 1993 a tan sólo 3,65 kgrs./hab./año. Como contrapartida, el mercado regional se resuelve como un mercado cautivo, desde el momento en que la entrada de limones desde otros puntos del país o desde el exterior se halla imposibilitada por la existencia de barreras fitosanitarias.

\section{Gráfico 2.}

\section{Destino de la producción limonera, en porcentajes sobre el total.}

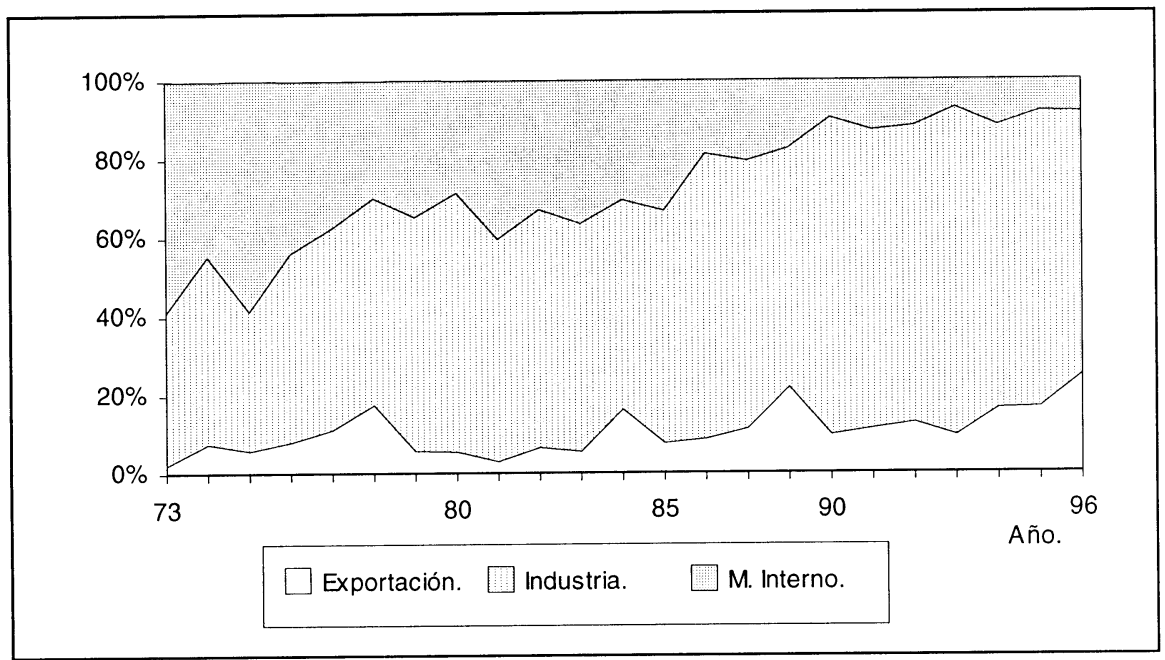

Fte: 1973/1988, S.E.A.G. 1989/1996, A.T.C.

Elaboración propia.

La última opción es el procesamiento industrial de la fruta. Es este destino el que mayoritariamente tiene la producción de las explotaciones más pequeñas, producción que, dada su baja calidad, no puede competir en otros mercados; su venta a granel a las industrias citrícolas, o la colocación de determinados contingentes en el mercado local, son generalmente 
las únicas alternativas que este estrato de productores tiene. Este mercado industrial ha experimentado un crecimiento muy importante (gráfico 2), dado que los niveles de demanda de zumos concentrados congelados tanto en el interior como en el exterior, y las buenas perspectivas de venta de otros derivados del limón (como aceites esenciales, cáscara deshidratada, etc.) han llevado al surgimiento en los últimos años de un gran número de industrias, que precisan cada vez mayores cantidades de materia prima; los procesos de integración vertical descendente a los que anteriormente hacíamos referencia deben ponerse en relación con estas crecientes exigencias de fruta para procesar, habiendo optado las industrias por incrementar sus plantaciones, en un intento por asegurarse unas cantidades mínimas de fruta al menor coste posible que rentabilicen la inversión.

Pero además, el acceso a estos diferentes mercados no depende exclusivamente de la calidad de la fruta obtenida -aunque, efectivamente, ésta se resuelva como un factor determinante-. Es así que los pequeños productores no cuentan con los medios económicos para colocar su producción directamente en el mercado nacional, menos aún en el externo; este último se resuelve como de difícil acceso incluso para los medianos productores, dado que la inversión necesaria para montar una empacadora propia es tan elevada que se resuelve como prohibitiva y, además, el umbral necesario para rentabilizarla es también muy elevado (200 has. como mínimo). Es aquí donde aparece la figura del intermediario; el más común es el propietario de una planta empacadora que a su propia producción une la adquirida a otros productores -siempre fruta de alta calidad-encargándose de su preparación y comercialización, proceso éste último en el que el acceso a la información es de crucial importancia. En este contexto en 1993 se creó la Comisión de Seguimiento a la Exportación, con el fin de evitar la aplicación de derechos compensatorios por parte de la Unión Europea. Integrada por representantes de los sectores privado y público, y habiendo establecido un sistema de intercambio de información con la Unión, una de las estrategias más empleadas es la prevención de la saturación puntual del mercado y la consecuente bajada de precios que determina la aplicación de los derechos, habiendo logrado ya en 1994 un aumento importante de las exportaciones a Europa a precios más altos que el año anterior (Dirección 1997, 18).

El precio que alcanza la fruta está determinado por el mercado en el que se vende, de manera que, ante las grandes diferencias existentes entre unos y otros, el destino de la producción se resuelve como determinante a la hora de cuantificar los ingresos de la explotación. A finales de la década de los ochenta, se estimaba que el precio relativo de la tonelada enviada al mercado externo equivalía a 2, a 1,5 para la venta en fresco en el mercado interno y tan sólo 0,4 cuando el destino era la fábrica (Salas 1989, 39). En la actualidad estas diferencias de precios según destino se siguen manteniendo, si no han aumentado; así, por un cajón de 18 kgrs. de limón exportado se pagaron 10,5 dólares de media en 1996 -575 dólares la tonelada- (Cohen $1997,4)$, precio que contrasta con los 362 \$/Tn cuando el destino era el mercado interno (precios en el Mercado Central de Buenos Aires según la S.E.A.G.), y especialmente con los 73 que, en promedio anual en lo que va de década, se pagaron en Tucumán por tonelada cuando el destino fue la fábrica, según la A.T.C.; no obstante, es preciso indicar que el precio pagado por las citrícolas está en estrecha relación con la cantidad de fruta entregada (superior cuanto mayor sea ésta), de ahí que, en determinados casos, los productores se agrupen de manera informal con el fin de, uniendo sus producciones, tratar de mejorar los bajos precios de partida. 
Si unimos estos precios a las posibilidades de venta en los distintos mercados que los estratos de productores tienen, y a las que ya hemos hecho referencia, resulta evidente que los ingresos provenientes de la venta son muy dispares entre uno y otro grupo, y que las posibilidades de diversificar la colocación de la producción entre los diferentes destinos se reducen conforme más pequeñas son las explotaciones.

La productividad de las explotaciones también está en relación con el número de hectáreas y, por tanto, con la capacidad económica del productor. Así, y basándonos en información obtenida a través de entrevistas a informantes calificados, los rendimientos por hectárea obtenidos por productores de hasta 30 hectáreas se situarían en torno a los 35.000 kgrs., mientras que aquellos productores con explotaciones de 100 has. o más obtendrían, en un promedio aproximado, $60.000 \mathrm{kgrs}$./ha. Estas diferencias en la productividad están en íntima relación con la cantidad de insumos empleados en la plantación; según Foguet (1992), una plantación bien llevada obtendría hasta 80 Tns. de limones por hectárea, entendiendo por tal aquella en la que se aplican agroquímicos en las cantidades apropiadas. No obstante, y al menos en los ochenta, existía una relación entre tamaño de la explotación y cantidad de fertilizante por planta empleado, tal y como se muestra en la tabla 2.

Tabla 2.

Cantidad, en kgrs./planta, de fertilizantes aplicados anualmente por tamaño de la explotación.

\begin{tabular}{cccc}
\hline Fertilizante. & $0-20$ has. & 20-50 has. & Más de 50 has. \\
\hline Urea & 1,350 & 1,530 & 1,700 \\
$15-15-15$ & 0,700 & 0,920 & 0,870 \\
Total & 2,050 & 2,450 & 3,220 \\
\hline
\end{tabular}

Fte: Lorente (1984, 13).

Valores que toman relieve si consideramos que para obtener una buena producción es necesario administrar al menos 1,6 kgrs. de urea por planta.

\section{COSTES DE PRODUCCIÓN.}

Como en cualquier actividad productiva, existe un determinado número de elementos necesarios para desempeñarla, cuyo valor se denomina coste de producción. Estos costes van a determinar en gran medida la rentabilidad de, en nuestro caso, las explotaciones limoneras, por lo que consideramos indispensable realizar un análisis de los mismos.

El coste de producción de una explotación limonera está compuesto por dos grupos fundamentales: uno, que engloba a los denominados "costes fijos" o "gastos indirectos", entre los que se encuetran los derivados del pago de impuestos y seguros, las amortizaciones -tanto de maquinaria como de la propia plantación-, intereses de diverso tipo, etc. El segundo grupo 
hace referencia a los "costes variables" o "gastos clirectos", originados en el empleo de fertilizantes, mano de obra, productos para el control de plagas, poda, riego, etc., elementos todos indispensables para la obtención de la producción y que, a diferencia de los anteriores, permiten cierto margen de maniobra al productor, recortándolos en momentos de estrechez o empleando más inputs cuando las disponibilidades económicas lo permiten.

En el cuadro que a continuación presentamos (tabla 3) se pueden observar los niveles estimados de gasto para explotaciones-tipo de diferentes dimensiones en la provincia de Tucumán. En él quedan recogidos con mayor nivel de detalle los gastos directos, mientras que el resto (amortizaciones, intereses e impuestos) han quedado reunidos bajo el epígrafe gastos indirectos, clado que se resuelven como gastos fijos e ineludibles.

Tabla 3.

Estimación a costes de explotaciones-tipo limoneras en la provincia de Tucumán.

\begin{tabular}{lccc}
\hline Rubro & 10 Has. (U\$S) & 30 Has. (U\$S) & 100 Has. (U\$S) \\
\hline Gastos directos & $\mathbf{2 1 . 6 4 1 , 8 3}$ & $\mathbf{6 8 . 3 7 5 , 4 5}$ & $\mathbf{3 7 4 . 6 4 8 , 5 3}$ \\
Control de malczals & $3.291,61$ & $9.874,82$ & $16.587,3$ \\
Trat. fitosanitario & $4.819,33$ & $14.457,98$ & $56.227,44$ \\
Fertilización & $1.712,74$ & $5.138,21$ & $37.627,54$ \\
Cosecha & $11.818,15$ & $38.904,44$ & $202.575,38$ \\
Poda & 0 & 0 & $10.130,87$ \\
Otros & 0 & 0 & 51.500 \\
\hline Gastos indirectos & $\mathbf{1 1 . 9 5 7 , 6 1}$ & $\mathbf{2 2 . 8 1 2 , 5 8}$ & $\mathbf{5 8 . 4 6 2 , 2 3}$ \\
\hline Total de gastos & $\mathbf{3 3 . 5 9 9 , 4 4}$ & $\mathbf{9 1 . 1 8 8 , 0 3}$ & $\mathbf{4 3 3 . 1 1 0 , 7 6}$ \\
\hline
\end{tabular}

Fte: Navarro et al. $(1997,9)$.

Vistos los costos teóricos de las plantaciones tucumanas, es preciso volver sobre los ingresos que obtendrían los dueños de las pequeñas explotaciones al comercializar su producción, para, de esta forma, acercarnos al conocimiento de su viabilidad y rentabilidad. Como ya se expresó, estos productores generalmente venden su producción a las industrias, como consecuencia de la baja calidad de la fruta, y por lo tanto dependen de los precios impuestos por ellas, precios que presentan una gran oscilación (gráfico 3). 
Gráfico 3.

Evolución de los precios pagados por tonelada de limón en las fábricas tucumanas.

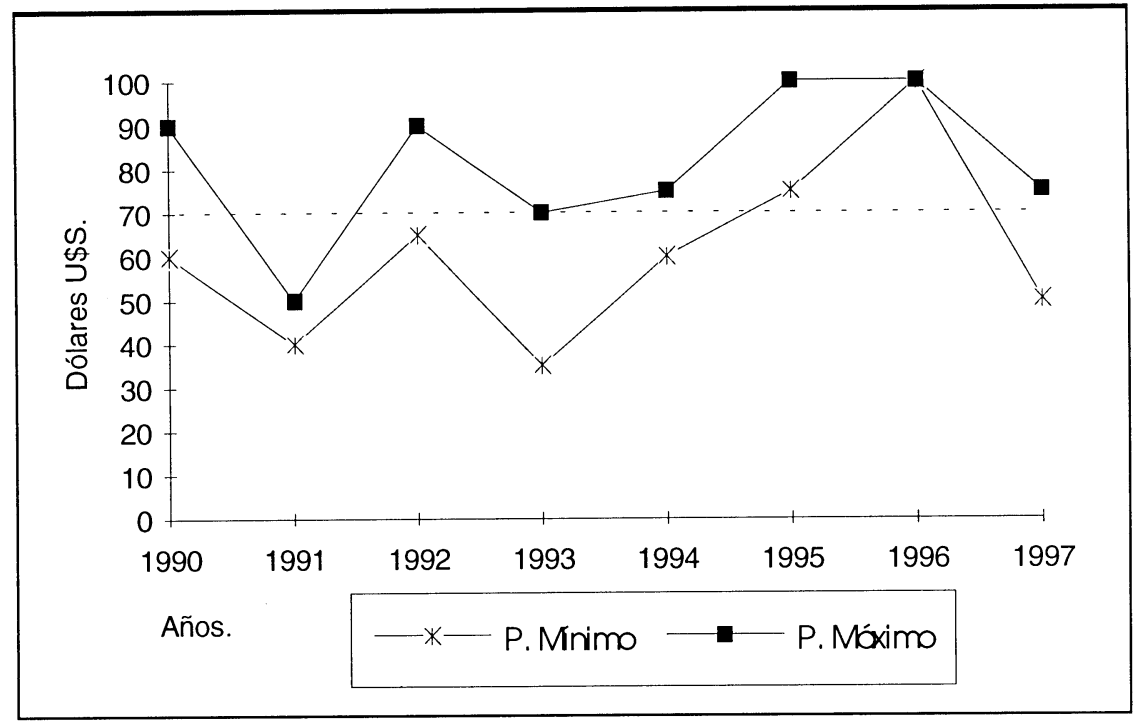

Ite: A.T.C. y Citrus Trade Famaillá.

En la situación teórica presentada en el ejemplo de 10 has., los autores estiman un rendimiento de $50 \mathrm{Tn}$./ha., ésto es, una producción de unas 500 toneladas en esta superficie. Si relacionamos dicha producción con los costes, veremos como sólo vendiendo la tonelada a un mínimo de 70 U\$S se podría cubrir los costos; este nivel de precios, tal y como se muestra en el gráfico anterior, no se alcanza todos los años, hecho que permitiría explicar en parte la tendencia a desaparecer de este tipo de explotaciones. Pero además hay que tener en cuenta que el modelo supone un nivel de producción que, en muchos casos, está muy por encima de los rendimientos actuales de las explotaciones de este tamaño; ello no vendría sino a empeorar sus perspectivas de supervivencia, desde el momento en que recortes en los costes directos, derivados de una disminución de los ingresos ${ }^{3}$, implican menor producción y de peor calidad, agravándose así la situación.

Contamos con algunas cifras que nos permitirían hacer una comparación muy general entre los costes de producción de 1 hectárea en España y en Tucumán. A la vista de las mismas se puede observar cómo en todos los rubros los valores son siempre superiores en el caso español (tabla 4).

3. Suponiendo una producción de 35 tns./ha. en las explotaciones con menos de 30 has., para cubrir los gastos generados por 10 has. tendrían que obtenerse $96 \mathrm{~J} \$ \$ \mathrm{~S} / \mathrm{tn}$. en fábrica; pero en realidad el productor obtiene, aplicáando la media de los precios pagados en lo que va de década, 25.50) U\$S por una cosechá que teóricamente le ha costaldo producir 33.60() . 


\section{Tabla 4.}

\section{Coste de producción en España y Argentina (Tucumán) de una hectárea de limón.}

\begin{tabular}{lcccc}
\hline & $\begin{array}{c}\text { España } \\
\text { Peseta }\end{array}$ & $\begin{array}{c}\text { Argentina } \\
\text { Peseta }\end{array}$ & $\begin{array}{c}\text { España } \\
\text { Dólar }\end{array}$ & $\begin{array}{c}\text { Argentina } \\
\text { Dólar }\end{array}$ \\
\hline Costes Variables/Gastos Directos & 390.208 & 302.960 & 2.782 & 2.164 \\
Costes Fijos/Gastos Indirectos & 192.277 & 167.440 & 1.373 & 1.196 \\
Total Coste de Producción & 582.485 & 470.400 & 4.161 & 3.360 \\
\hline
\end{tabular}

Fuente: Navarro, H., et al. (1997:9), Caballero, P., et al. (1992), cit. por A.T.C. (1993, 24)

Nota: 1 dólar equivale a 140 pesetas.

Las mayores diferencias están en los gastos directos, y son debidas, fundamentalmente, a los costes de la mano de obra y riego. En el caso tucumano las mayores precipitaciones (entre 700 y $1.000 \mathrm{mms}$. anuales, concentradas en el verano) permiten producir (aunque no en óptimas condiciones) sin riego; pero en este aspecto también hay diferencias según el tamaño de la explotación. Así, y si bien en las mayores el riego (mayoritariamente por gravitación y aspersión) se está extendiendo cada vez más, en las pequeñas es una práctica que normalmente no se lleva a cabo. En contraste, en el caso español el riego se resuelve como imprescindible a la hora de obtener cosechas medianamente aceptables, dadas las menores cantidades de precipitaciones.

La mano de obra presenta diferencias tanto en el número de jornales como en su precio. En el valle del Gualdalhorce se llegan a emplear hasta 37 jornales por hectárea tan sólo en las labores culturales (Larrubia 1996, 162), cifra que contrasta con los 7 que, en estas mismas tareas, se emplean en Tucumán (Lizárraga 1994, 13). Esta disparidad viene explicada porque en Málaga la poda (25 jornales) se hace de forma manual, mientras que en Tucumán, o se practica esporádicamente en las explotaciones menores, o bien se lleva a cabo de forma mecanizada en las mayores. Por otro lado, el coste del jornal en Málaga asciende a 3.000 ptas. (unos $21 \mathrm{U} \$ \mathrm{~S}$ ), mientras que el jornalero tucumano tan sólo recibe, en el mejor de los casos 12 U\$S (A.T.C. 1996, 26), unas 1.700 ptas. Esto nos lleva a un coste medio de 118.000 ptas. (840 U\$S) en Málaga, frente a tan sólo 84 U\$S (12.000 ptas.) en Tucumán; diferencias que, aún ausmiendo que parte del trabajo en las explotaciones del Valle del Guadalhorce sea familiar, son tan elevadas que influyen decisivamente en el coste general de la explotación.

El resto de particlas incluíclas en los gastos directos presenta también diferencias, aunque de menor cuantía. El mayor gasto en productos fitosanitarios generado en las explotaciones tucumanas (en España el tratamiento fitosanitario es menos costoso, dado que su clima más seco no favorece el desarrollo de determinadas plagas -Caram 1994, 16-) viene compensado por una menor inversión en fertilizantes en las explotaciones más pequeñas, lo que va en detrimento de la productividad.

Por tanto, la producción española por hectárea resulta más cara que la tucumana; no obstante, son mayoría en el agro español las explotaciones limoneras menores a una hectárea, 
mientras que en Tucumán éstas prácticamente han desaparecido. Esta aparente contradicción se explica por varios factores. Uno de ellos es la existencia de "Tasas de Compensación" aplicadas por la Unión Europea. Estas tasas gravan las producciones foráneas vendidas a precios inferiores al precio de referencia, protegiendo así la venta de fruta fresca por parte de los países productores de limón de la Comunidad. Tasas que, en algunos años, pueden ser superiores a los propios precios FOB (Dirección 1997, 17).

Por otro lado, el agricultor español está protegido, bajo ciertas condiciones, por la existencia de un precio de intervención que le asegura al menos unos ingresos mínimos cuando comercialize su producción a través de una cooperativa, aunque tan sólo hasta cierto nivel.

No obstante, la bajada de los precios que el limón ha experimentado en los últimos años en relación a los existentes a finales de la década de los setenta, ha hecho que un determinado número de productores se dedique a la actividad citrícola de forma parcial en una explotación que, al menos en el Valle del Guadalhorce, no se resuelve como antieconómica en el medio plazo; vendiendo el producto a unos precios entre 30 y 35 ptas. el kilo, el margen neto de beneficio para el agricultor será de entre 720.000 y 900.000 ptas./ha. (Larrubia 1998, 162). En contraste, y como vimos, el pequeño productor tucumano, ante lo desfavorable de la relación precio de venta/coste de insumos y la ausencia de medidas de pretección, ve cómo sus hectáreas, lejos de dar beneficio, acumulan pérdidas, por lo que se ve obligado a abandonar la actividad.

\section{CONCLUSIONES}

El importante crecimiento experimentado por la actividad limonera tucumana ha estado condicionado por la demanda y exigencias de los diferentes mercados, conduciendo por norma general a una mayor competitividad de las explotaciones. En dicho proceso fueron las pequeñas superficies las que no pudieron acceder a los mercados más rentables por la imposibilidad de acometer las inversiones necesarias para mejorar la calidad de su fruta, viéndose así limitadas a destinar sus limones a la industria, donde los precios alcanzan los menores valores de cotización por este producto. Esta situación de libre mercado sin duda ha incentivado la eficiencia en la producción, al tiempo que, paralelamente, ha conducido a un paulatino empobrecimiento y desaparición de los pequeños productores limoneros, aumentando así una vez más las diferencias entre los actores sociales intervinientes en esta agroindustria.

Así, ante la alta competitividad existente en el mercado mundial del limón, las pequeñas explotaciones tan sólo pueden perdurar bajo condiciones muy concretas; es el caso de las españolas, que al quedar inmersas en uno de los bloques económicos más importantes, la Comunidad Europea, se ven amparadas por su política proteccionista y los subsidios, permi-

tiendo que aún subsistan explotaciones de una hectárea, situación que, en la actualidad, resulta impensable en el contexto tucumano. 


\section{BIBLIOGRAFÍA.}

AMIGO, J.L. et al. (1994): Estudio de competitividad agropecuaria y agroindustrial. Subsector cítricos. Ed. Instituto Interamericano de Cooperación para la Agricultura, Buenos Aires. 173 pags.

ASOCIACIÓN TUCUMANA DE CITRUS (1994): Noticiero N² 29. Ed. A.T.C. San Miguel de Tucumán.

CARAM, E. (1994): La organización del comercio internacional de frutos cítricos. (estructuras, conductas y resultados). Tesis de magister, inédita. 199 pags.

COHEN, G. (1997): Panorama general de la citricultura argentina. S.E.A.G., Buenos Aires. 5 pags.

FOGUET, J. (1992): "Provincia de Tucumán (Informe citrícola)", en XVJornada citrícola nacional. Ed., Concordia.

LARRUBIA VARGAS, R. (1988): "Situación y problemática de la producción y comercialización citrícola en el Valle del Guadalhorce", en Baetica, Estudios de Arte, Geografía e Historia $N^{\circ}$ 11. Ed. Facultad de Filosofía y Letras, Universidad de Málaga, Málaga, 41-69.

LARRUBIA VARGAS, R. (1994): Producción y comercialización de los cítricos en la provincia de Málaga. Biblioteca Popular Malagueña No 66. Ed. Servicio de Publicaciones de la Diputación Provincial de Málaga, Málaga. 165 págs.

LARRUBIA VARGAS, R. (1996): “Resultados económicos de las producciones agrarias. Evolución de los precios, costos de producción y márgenes de comercialización", en Baetica, Estudios de Arte, Geografía e Historia No 18. Ed. Facultad de Filosofía y Letras, Universidad de Málaga, Málaga, 129-177.

LORENTE, F. (1984): Organización, productividad y rentabilidad de las explotaciones limoneras en la provincia deTucumán. Mimeo, 38 pags.

NAVARRO, H.R. et al. (1997): Indicadores económicos en modelos de fincas limoneras de la provincia de Tucumán. Mimeo, 12 pags.

OCAÑA OCAÑA, C., y LARRUBIA VARGAS, R. (1993): Agricultura y espacio metropolitano: Málaga y el Bajo Guadalhorce. Textos mínimos $\mathrm{N}^{\circ} 22$. Ed. Secretariado de Publicaciones e Intercambio Científico de la Universidad de Málaga, Málaga. 169 págs.

SALAS OROÑO, J. (1989): "Población", en I.P.D.U. (1988): Red de centros de servicios rurales de la provincia de Tucumán. Ed. Universidad Nacional de Tucumán, Tucumán, 32-42. 